\title{
Relations entre le niveau d'ingestion d'énergie et quelques paramètres sanguins chez le chevreau au cours des 2 semaines suivant le sevrage
}

\author{
P Bas, P Morand-Fehr, A Rouzeau, D Sauvant \\ Station de Nutrition et Alimentation (INRA) de I'INA-PG, \\ 16, rue C Bernard, 75231 Paris Cedex 05, France
}

\begin{abstract}
Summary - Eighteen male kids were fed ad libitum hay and concentrate from weaning at 4, 6 or 8 wh of age. Metabolizable energy intake $\mathrm{kg}$ of live weight ${ }^{0.75}$ was more closely related to $\beta$ hydroxybutyrate than to non-esterified fatty acids or glycemia during the first post-weaning wk, but it was the contrary during the next one.
\end{abstract}

Introduction - Les études sur l'évolution de l'état nutritionnel énergétique des ruminants juste après le sevrage sont rares (Kouamé et al, 1984). A notre connaissance aucune étude n'a été réalisée sur chevreaux. Nous avons donc analysé les relations entre l'ingestion d'énergie métabolisable et les paramètres sanguins aussitôt après le sevrage des chevreaux.

Matériel et Méthodes - Dix-huit chevreaux mâles de race Alpine, élevés en case individuelle, sont alimentés à volonté avec du lait de remplacement $(19,5 \%$ de matières grasses, $24,2 \%$ de matières azotées (MAT) dans la matière sèche (MS); $4700 \mathrm{kcal}$ d'énergie métabolisable (EM)/kg MS) jusqu'au sevrage brutal pratiqué à 4,6 ou 8 semaines. Depuis la semaine précédant le sevrage, les chevreaux disposent en permanence d'eau, de foin de luzerne (17,1\% MAT/MS; $2100 \mathrm{kcal}$ EM/kg MS) et d'un aliment concentré $(19,4 \%$ MAT/MS; $3000 \mathrm{kcal}$ EM/kg MS). Les consommations individuelles de chaque aliment sont enregistrées chaque jour. Les valeurs d'EM des aliments d'allaitement et celles des aliments solides ont été relevées dans les Tables de I'INRA (1978). Les acides gras non estérifiés (AGNE), le $\beta$ hydroxybutyrate (BHB) et le glucose sont dosés dans le plasma des échantillons de sang prélevés, la veille du sevrage $(J-1)$ à $8 \mathrm{~h}$ du matin puis $2,5,7$ et $14 \mathrm{j}$ après ( $\mathrm{J} 2 \mathrm{a} \mathrm{J} 14)$.
Résultats et Discussion - Avant le sevrage $(J-1)$, les concentrations de ces 3 métabolites sanguins ne sont significativement influencées ni par l'âge des chevreaux ni par la quantité d'EM ingérée/ $\mathrm{kg}^{0,75}\left(E M I / P{ }^{0,75}\right)$, la veille du prélèvement de sang (tableau I). Le sevrage brutal induit une augmentation importante $(\approx 5$ fois) des AGNE et du BHB mais seulement une baisse modérée $(-20 \%)$ de la glycémie. De J2 à J14, seule la concentration des AGNE est redevenue comparable à celle de $\mathrm{J}-1$. $\mathrm{A} \mathrm{J} 2$, le BHB est le plus élevé chez les chevreaux sevrés à 4 semaines $(P<0,05)$ et le plus bas chez ceux sevrés à 6 semaines. Cette différence s'estompe lorsque la comparaison est effectuée à même EMI/P 0.75. L'âge au sevrage n'influence pas la concentration des autres métabolites de J2 à J14. A J2, la liaison entre l'EMI/P 0.75 et le BHB est plus étroite que celle entre l'EMI/P 0,75 et les AGNE, peut être à cause d'une plus grande susceptibilité des AGNE à varier sous l'action de facteurs d'origine extrinsèque (Bowden, 1971). Cette dernière relation n'est significative que lorsque les AGNE sont exprimés sous forme logarithmique ce qui confirme les résultats de Giger et Sauvant (1982). Ce fait peut ré- 
Tableau I. Evolution de la concentration des métabolites sanguins $(\bar{x})$ el de leurs coefficients de corrélation (r) avec l'énergie métabolisable ingérée par $\mathrm{kg}$ de poids métabolique, $\left(E M / / P^{0.75}\right)$ la veille du prélèvement de sang.

\begin{tabular}{|c|c|c|c|c|c|c|}
\hline & & $J-1$ & $J 2$ & $J 5$ & $J 7$ & $J 14$ \\
\hline $\begin{array}{l}\text { Glycémie } \\
(\mathrm{g} / \mathrm{l})\end{array}$ & $\stackrel{x}{r}$ & $\begin{array}{l}1,20^{\mathrm{a}} \\
0,279\end{array}$ & $\begin{array}{l}0,93^{b} \\
0,018\end{array}$ & $\begin{array}{l}0,93^{\mathrm{b}} \\
0,704^{* *}\end{array}$ & $\begin{array}{l}0,92^{b} \\
0,530^{*}\end{array}$ & $\begin{array}{l}0,89^{b} \\
0,641^{* *}\end{array}$ \\
\hline $\begin{array}{l}\text { AGNE } \\
(\mu \mathrm{mol} / 1)\end{array}$ & $\begin{array}{l}x \\
r \\
r i n\end{array}$ & $\begin{array}{l}133^{a} \\
0,209 \\
0,326\end{array}$ & $\begin{array}{l}596^{b} \\
-0,320 \\
-0,543^{*}\end{array}$ & $\begin{array}{l}491^{b} \\
-0,604^{* *} \\
-0,619^{* *}\end{array}$ & $\begin{array}{l}332^{c} \\
-0,600^{* *} \\
-0,646^{* *}\end{array}$ & $\begin{array}{l}152^{a} \\
-0,647^{* *} \\
-0,679^{* *}\end{array}$ \\
\hline $\begin{array}{l}\text { BHB } \\
(\mathrm{mg} / 1)\end{array}$ & $\begin{array}{l}x \\
r \\
r l n\end{array}$ & $\begin{array}{l}5,7^{a} \\
0,008 \\
0,001\end{array}$ & $\begin{array}{l}32,2^{b} \\
-0,594^{* *} \\
-0,501^{*}\end{array}$ & $\begin{array}{l}38,0^{\mathrm{b}} \\
-0,709^{* *} \\
-0,652^{* *}\end{array}$ & $\begin{array}{l}32,4^{b} \\
-0,828^{* *} \\
-0,748^{* *}\end{array}$ & $\begin{array}{c}23,9 c \\
-0,109 \\
-0,172\end{array}$ \\
\hline $\begin{array}{l}E M / / P^{0,75} \\
\left(\mathrm{kcal} / \mathrm{kg} P^{0,75}\right)\end{array}$ & $\begin{array}{l}\vec{x} \\
s\end{array}$ & $\begin{array}{r}275 \\
35\end{array}$ & $\begin{array}{l}44 \\
33\end{array}$ & $\begin{array}{l}63 \\
47\end{array}$ & $\begin{array}{l}81 \\
49\end{array}$ & $\begin{array}{r}127 \\
58\end{array}$ \\
\hline
\end{tabular}

$\mathrm{J}$ : date du prélèvement de sang par rapport au sevrage (en j); $r n$ : coelficients de corrélations des concentrations des métabolites sanguins exprimées sous forme logarithmique; $s$ : écart type; ${ }^{*}: P<0,05,{ }^{* *} P<0,01$; les valeurs non affectées de lettres communes sont signifcativement différentes à $P<0,05$.

sulter d'une saturation relative de la capacité de leur prélèvement et/ou d'oxydation par les tissus. A J2, la glycémie paraît indépendante de l'EMI/P ${ }^{0.75}$. Ces trois métabolites sont significativement corrélés à I'EMI/P 0,75 à $\mathrm{J} 5$ et $\mathrm{J} 7$, mais à $\mathrm{J} 14$ la liaison entre le BHB et l'EMI/P 0,75 s'est fortement atténuée en raison probablement de l'accroissement du flux entrant de BHB d'origine digestive.

Pour l'ensemble de la période postsevrage étudiée, les variations d'énergie ingérée sont le mieux expliquées par les AGNE ou par les AGNE et le BHB: EMI/ $P 0,75(\mathrm{kcal} / \mathrm{j} / \mathrm{kg} \quad P .0,75)=-42,2$ AGNE (Ln $\mu \mathrm{mol} / \mathrm{l})-0,359 \mathrm{BHB}(\mathrm{mg} / \mathrm{l})+330$; (écart type résiduel $(s r)=24,5, r=-0,928$, $n=72$ ). Bien que les prélèvements de sang n'aient pas été effectués après une période de jeûne et que l'EM ingérée n'ait pas été mesurée, ses variations sont néanmoins bien expliquées à partir de ces métabolites sanguins. En incluant les prélèvements de $\mathrm{J}-1(n=90)$, sr augmente de plus de $100 \%$ probablement à cause du flux d'AGNE provenant les lipides ingérés et du faible niveau de la lipolyse.

En conclusion, les variations plasmatiques des AGNE, du BHB et du glucose ne sont étroitement liées à celles de l'EMI que lorsque le chevreau est en état de sousnutrition. Juste après le sevrage, la chute d'ingestion de l'EMI est la mieux expliquée par le BHB puis ultérieurement par les AGNE.

Bowden DM (1971) Can J Anim Sci 51, 1-13

Giger S, Sauvant D (1982) Ann Zootech 31, 149-160

INRA (1978) Alimentation des Ruminants. INRA Publ, Versailles, 519-555

Kouamé KG, Troccon JL, Patureau-Mirand $P$, Journet M, Pion R (1984) Ann Zootech 33, 427-444 